\title{
COMPRESSIVE SENSING FOR THROUGH WALL RADAR IMAGING OF STATIONARY SCENES USING ARBITRARY DATA MEASUREMENTS
}

\author{
Eva Lagunas ${ }^{1}$, Moeness G. Amin ${ }^{2}$, Fauzia Ahmad ${ }^{2}$, and Montse Nájar ${ }^{1}$ \\ ${ }^{1}$ Universitat Politècnica de Catalunya (UPC), Barcelona, Spain \\ ${ }^{2}$ Radar Imaging Lab, Center for Advanced Communications \\ Villanova University, Villanova, PA 19085, USA
}

\begin{abstract}
In this paper, we deal with removal of wall EM reflections prior to image reconstruction using step-frequency radars. The goal is to enable behind-the-wall target detection and localization from reduced data measurements. In the underlying problem, few frequency observations are available and they differ from one antenna position to another in a SAR imaging system. Because of using a different set of frequencies for different antennas, direct applications of wall clutter mitigation methods, such as subspace and spatial filtering, prove ineffective. To provide these methods with the response measured at the same set of frequencies, a compressive sensing approach is used to reconstruct the range profiles. We use prior knowledge of the wall standoff distance to speed up the convergence of the Orthogonal Matching Pursuit for sparse data reconstruction.
\end{abstract}

\section{INTRODUCTION}

One of the primary objectives of through-the-wall radar imaging (TWRI) is to detect and recognize objects behind walls using microwave signals [1-5]. In TWRI, the backprojection method is typically employed for image formation. Recently, it has been shown that compressive sensing (CS) and $l_{1}$ norm reconstruction techniques can be applied, in lieu of backprojection. In so doing, significant savings in acquisition time can be achieved. Additionally, producing an image of the indoor scene using few observations can be logistically important, as some of the measurements can be difficult, or impossible to attain. For instance, EM transmission at some antenna locations in a SAR system can be blocked by natural or manmade surrounding obstacles. On the other hand, some individual frequencies or frequency subbands may be unavailable due to competing wireless services or intentional interferences.

Most papers apply CS to TWRI assuming prior complete removal of the wall EM returns. Without this assumption, strong clutter introduced by the wall which extends along the range dimension makes the scene far from

This work was supported by ONR under grants N00014-11-1-0576 and N0014-10-1-0455.

E. Lagunas is partially supported by the fellowship FI-DGR-2011. being sparse and thus prevents the application of CS. Attempting to remove the wall reflections from the received data by change detection cannot be performed for stationary scenes. This is because the subtraction of consecutive imaging results would eliminate both target and clutter. For this type of scenes, techniques to remove the front wall EM returns without diminishing the target have been devised [6-10]. These approaches were originally introduced to work on all data observations [6-9], and were later shown to be equally effective under partial data observations, thereby permitting the application of CS for sparse scene reconstruction [10]. More specifically, direct applications of wall clutter mitigation techniques, such as spatial filtering [9] and subspace projection [8], were shown to be effective in [10], provided that the same reduced set of frequencies or time samples were used at each antenna position. Relaxation of this key condition would lead to degradation in wall clutter mitigation performance. In the case of spatial filtering, using different frequencies at different antenna positions would generate different wall reflection phase returns and, subsequently, deprive the notch filter from the fundamental assumption of having the wall clutter residing at the zero spatial frequency. For the subspace method, removing the condition of using the same frequencies across the array aperture would increase the wall subspace dimension to the extent that the target subspace will not be clearly identified, rendering orthogonal subspace projection ineffective.

This paper combines wall mitigation techniques with CS for the situations where a different set of random data samples, in time or frequency, are collected at different antennas. This is made possible by first reconstructing the range profile, which is based on $l_{1}$ norm minimization. Then, the data of the missing frequencies can be obtained by taking the FFT of the reconstructed range profile at each antenna. A similar approach was adapted in [11]; however, it required the fundamental condition of prior removal of wall EM scattering to be satisfied. Once the phase returns corresponding to all original frequencies are estimated, wall mitigation can proceed using spatial filtering, the subspace approach, or any other conventional wall mitigation method.

The reconstruction of the range profiles is performed using a modification of the Orthogonal Matching Pursuit (OMP) algorithm. Since the target is behind the wall, the 
OMP can be modified such that the iterations corresponding to the range up to the wall can be bypassed. This allows a quicker inclusion of the target into the reconstruction algorithm. We compare OMP with the modified OMP and show the abilities as well as the challenges of performing TWRI with arbitrary data measurements.

The remainder of this paper is organized as follows. Next section provides the TWRI signal model and reviews the wall mitigation approaches presented in [9] and [8]. Section 3, presents the proposed compressive sensing approach used to reconstruct the range profiles along with the modified OMP. In Sections 4 and 5, respective results for simulated and experimental data are discussed. Finally, Section 6 states the conclusions.

\section{SIGNAL MODEL AND WALL MITIGATION TECHNIQUES}

We first describe the signal model for through-the-wall propagation in the presence of a homogeneous wall and then discuss the wall mitigation approaches presented in [9] and [8].

\subsection{Through-the-Wall Signal Model}

Consider an $N$-element line array of transceivers parallel to a homogeneous wall. Let the $n$th transceiver illuminate the scene with a stepped-frequency signal of $M$ frequencies. The reflection by the wall and any targets in the scene are measured only at the same transceiver location. Assuming the scene contains $P$ point targets, the signal measured at the $n$th transceiver using the $m$ th frequency is given by,

$y(m, n)=\sigma_{w} e^{-j\left(\omega_{0}+m \Delta_{\omega}\right) \tau_{w}}+\sum_{p=0}^{P-1} \sigma_{p} e^{-j\left(\omega_{0}+m \Delta_{\omega}\right) \tau_{p, n}}$

where $\omega_{0}$ is the lowest frequency in the bandwidth spanned by the stepped-frequency signal, $\Delta_{\omega}$ is the frequency step size, $\sigma_{w}$ is the wall complex reflectivity, $\sigma_{p}$ is the complex reflectivity of the $p$ th target, $\tau_{w}$ is the two-way traveling time of the signal from the $n$th antenna to the wall and $\tau_{p, n}$ is the two-way traveling time from the $n$th antenna to the target. For through-the-wall propagation, $\tau_{p, n}$ will comprise the components corresponding to traveling distances before, through, and after the wall [12].

\subsection{Spatial Filtering Approach}

From (1), we note that $\tau_{w}$ does not vary with the sensor location since the array is parallel to the wall. This implies that the first term in (1) assumes the same value across the array aperture. Unlike $\tau_{w}$, the time delay $\tau_{p, n}$ in (1) is different for each antenna location, since the signal path from the antenna to the target varies from one antenna to the other. For the $m$ th frequency, the received signal is a function of $n$ via the variable $\tau_{p, n}$. Therefore, we can rewrite (1) as,

$$
y_{\omega_{m}}(n)=v_{\omega_{m}}+\sum_{p=0}^{P-1} u_{p, \omega_{m}}(n)
$$

where,

$$
\begin{gathered}
v_{\omega_{m}}=\sigma_{w} e^{-j\left(\omega_{0}+m \Delta_{\omega}\right) \tau_{w}} \\
u_{\omega_{m}}(n)=\sigma_{p} e^{-j\left(\omega_{0}+m \Delta_{\omega}\right) \tau_{p, n}}
\end{gathered}
$$

Thus, separating wall reflections from target reflections amounts to basically separating constant from non-constant valued signals across the antennas. This can be performed by applying a proper spatial filter [9]. In its simplest form, the spatial filter, which notches out the zero spatial frequency component, can be implemented as the subtraction of the average of the radar return across the antennas. That is,

$$
\tilde{y}_{\omega_{m}}(n)=y_{\omega_{m}}(n)-\frac{1}{N} \sum_{n=0}^{N-1} y_{\omega_{m}}(n)
$$

The filtered data will have little or no contribution from the wall reflections.

\subsection{Subspace Approach}

The signals received by the $N$ antennas at the $M$ frequencies are arranged into an $M \times N$ matrix, $\mathbf{Y}$,

$$
\mathbf{Y}=\left[\begin{array}{lllll}
\mathbf{y}_{0} & \cdots & \mathbf{y}_{n} & \cdots & \mathbf{y}_{N-1}
\end{array}\right]
$$

where $\mathbf{y}_{n}$ is the $M \times 1$ vector containing the steppedfrequency signal received by the $n$th antenna,

$$
\mathbf{y}_{n}=\left[\begin{array}{lllll}
y(0, n) & \cdots & y(m, n) \quad \cdots & y(M-1, n)
\end{array}\right]^{T} .
$$

The eigen-structure of the imaged scene is obtained by performing the Singular Value Decomposition (SVD) of $\mathbf{Y}$,

$$
\mathbf{Y}=\mathbf{U} \boldsymbol{\Lambda} \mathbf{V}^{H}
$$

where $\mathrm{H}$ denotes the Hermitian transpose, $\mathbf{U}$ and $\mathbf{V}$ are unitary matrices containing the left and right singular vectors, respectively, and $\boldsymbol{\Lambda}$ is a diagonal matrix containing the singular values $\lambda_{1}, \lambda_{2}, \ldots, \lambda_{N}$ in decreasing order, i.e., $\lambda_{1} \geq \lambda_{2} \geq \ldots \geq \lambda_{N}$. The SVD method assumes that the wall returns and the target reflections lie in different subspaces. Therefore, the first $K$ dominant singular vectors of the $\mathbf{Y}$ matrix are used to construct the wall subspace,

$$
\mathbf{S}_{\text {wall }}=\sum_{i=1}^{K} \mathbf{u}_{i} \mathbf{v}_{i}^{H}
$$

In general, the dimension of the wall subspace $K$ depends on the degree of heterogeneity of the wall. For homogeneous walls, $K=1$. The subspace orthogonal to the wall subspace is,

$$
\mathbf{S}_{\text {wall }}^{\perp}=\mathbf{I}-\mathbf{S}_{\text {wall }} \mathbf{S}_{\text {wall }}^{H}
$$

where $\mathbf{I}$ is the Identity matrix. To mitigate the wall returns, the data matrix $\mathbf{Y}$ is projected on the orthogonal subspace,

$$
\tilde{\mathbf{Y}}=\mathbf{S}_{\text {wall }}^{\perp} \mathbf{Y}
$$




\section{CS FOR TWRI}

In this section, CS is applied to the data samples of the radar return measured at each antenna location separately. Assuming the range of interest is divided into $M$ equally spaced gates, we obtain a linear matrix equation relating the $n$th received signal and the target locations as

$$
\mathbf{y}_{n}=\Psi \mathbf{s}_{n}
$$

where $\mathbf{s}_{n}$ is the discrete range profile at the $n$-th antenna location, $\mathbf{y}_{n}$ is the measured data corresponding to all $M$ frequencies at the $n$th antenna, and the $l$ th column of $\Psi$ is defined as,

$$
\boldsymbol{\psi}_{l}=\left[\begin{array}{lll}
e^{-j \omega_{0} \frac{2 l d}{c}} & \cdots & e^{-j\left(\omega_{0}+(M-1) \Delta_{\omega}\right) \frac{2 l d}{c}}
\end{array}\right]^{T}
$$

with $d=\frac{c}{2\left(\omega_{M-1}-\omega_{0}\right)}$ and $c$ is the speed of the light. Note that the dimension of $\mathbf{s}_{n}$ is equal to the number of range gates, whereas the dimension of $\mathbf{y}_{n}$ is equal to the number of frequencies.

Consider $\tilde{\mathbf{y}}_{n}$ which is a vector of length $Q(<<M)$ consisting of elements chosen from $\mathbf{y}_{n}$ as follows,

$$
\tilde{\mathbf{y}}_{n}=\boldsymbol{\Phi}_{n} \mathbf{y}_{n}=\boldsymbol{\Phi}_{n} \boldsymbol{\Psi} \mathbf{s}_{n}
$$

where $\boldsymbol{\Phi}_{n}$ is the $Q \times M$ measurement matrix constructed by randomly selecting $Q$ rows of an $M \times M$ identity matrix. The number of measurements $Q$ required to achieve successful CS reconstruction depends on the coherence between $\boldsymbol{\Phi}$ and $\boldsymbol{\Psi}$. For the problem at hand, $\boldsymbol{\Phi}$ is the canonical basis and $\boldsymbol{\Psi}$ is similar to the Fourier basis, which have been shown to exhibit maximal incoherence [13]. In general, assuming that the sparse signal $\mathbf{s}_{n}$ has $s$ dominant components, the number of linear measurements required to recover $\mathbf{s}_{n}$ is given by $Q=O(s \log (M / s))$ [14].

Given $\tilde{\mathbf{y}}_{n}$, we can recover $\mathbf{s}_{n}$ by solving the following equation,

$$
\hat{\mathbf{s}}_{n}=\arg \min _{\mathbf{s}_{n}}\left\|\mathbf{s}_{n}\right\|_{l_{1}} \quad \text { subject to } \quad \tilde{\mathbf{y}}_{n} \approx \mathbf{D}_{n} \mathbf{s}_{n}
$$

where $\mathbf{D}_{n}=\boldsymbol{\Phi}_{n} \boldsymbol{\Psi}$. Once the range profile $\hat{\mathbf{s}}_{n}$ has been obtained, we can recover all $M$ frequency measurements at the $n$th antenna location as $\hat{\mathbf{y}}_{n}=\mathbf{\Psi} \hat{\mathbf{s}}_{n}$. Then, either one of the two wall mitigation methods described in Section 2 can be readily applied. Finally, backprojection can be used to reconstruct the image of the scene. However, since the wall clutter has been suppressed, an $l_{1}$ minimization based scene reconstruction can be applied, in lieu of backprojection, to improve the target-to-clutter ratio [10], [15].

A variety of methods are available in the literature to solve the optimization problem in (15). The $l_{1}$-minimization is a convex problem and can be recast as a linear program (LP). This is the foundation for the Basis Pursuit (BP) technique. Alternatively, greedy methods, known as Matching Pursuit (MP), can be used to solve (15) iteratively. As BP is computationally expensive, we chose to solve (15) using the MP. More specifically, we use the OMP, which is known to provide a fast and easy to implement solution. Moreover, OMP is better suited when
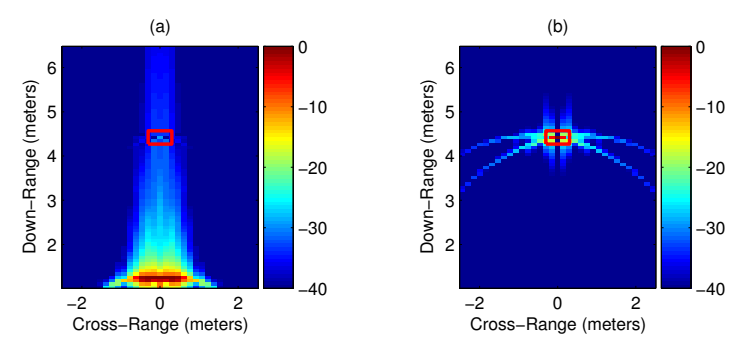

Fig. 1. TWRI images of the simulated scene: (a) no preprocessing, (b) after background subtraction.

frequency measurements are used [16]. However, OMP does not take into account the specificities of the throughthe-wall radar problem in (15). At each iteration, the OMP selects the column of $\mathbf{D}$ best correlated with the residual part of the signal. Then, it produces a new approximation by projecting the signal onto the dictionary elements that have already been selected. As the wall returns are much stronger than the target reflections, the first few iterations of the OMP always select range gates corresponding to the wall response. Taking this fact into account, we modify the first iteration of the OMP to select a set of range gates in the neighborhood of the wall. This modification reduces the number of iterations, thereby saving computational time. This saving is particularly important in the underlying problem since equation (15) needs to be solved for each antenna location.

\section{SIMULATION RESULTS}

In this section, we evaluate the performance of the proposed scheme using synthesized data. A stepped-frequency signal covering the $1-3 \mathrm{GHz}$ frequency band with a step size of $2.75 \mathrm{MHz}$ was employed, providing a range resolution $d$ of $0.075 \mathrm{~m}$. A 67 -element line array with an inter-element spacing of $0.0187 \mathrm{~m}$, located along the $\mathrm{x}$ axis, was used for imaging. The scene consisted of a single point target located at $(0,4.43) \mathrm{m}$ and a homogeneous wall located at a downrange of $1.28 \mathrm{~m}$. The wall return is assumed to be $40 \mathrm{~dB}$ higher than the target return. The region to be imaged is chosen to be $5.4 \mathrm{~m}$ (down-range) $\times 4.9 \mathrm{~m}$ (cross-range) centered at $(0,2.7) \mathrm{m}$ and is divided into $73 \times 33$ pixels, respectively.

Fig. 1(a) shows the image corresponding to the simulated scene obtained with conventional backprojection applied directly to the full raw dataset. The red rectangle depicted in the figure indicates the true position of the target. In this figure and all subsequent figures in this paper, we plot the image intensity with the maximum intensity value in each image normalized to OdB. Fig. 1(b) shows the image after background subtraction. The target is now clearly visible. Since access to the background scene is not available in practice, wall mitigation techniques must be applied, as a preprocessing step, to unmask the targets otherwise obscured by the strong wall reflections.

Fig. 2 depicts the backprojection images of the scene obtained after applying the spatial filter (Fig. 2(a)) and 

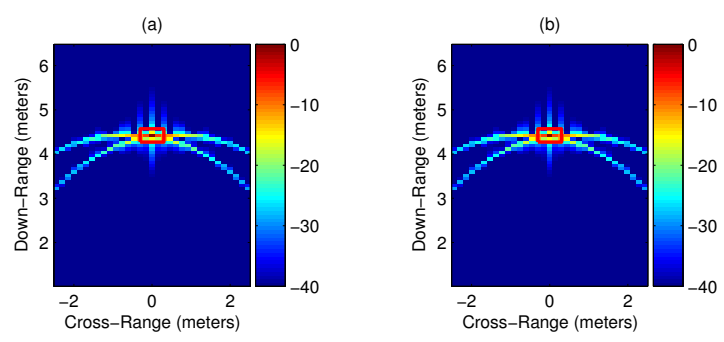

Fig. 2. Wall mitigation techniques with full data: (a) Spatial filtering, (b) SVD approach. (a)

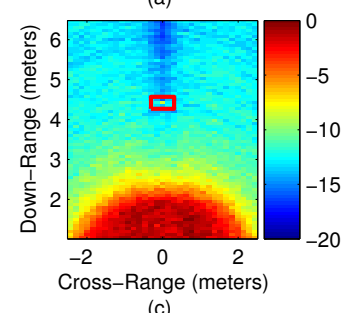

(c)

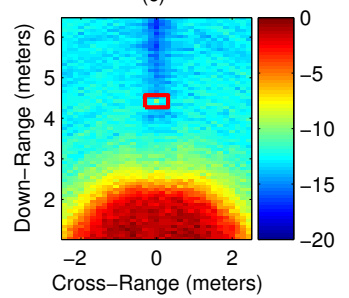

(b)
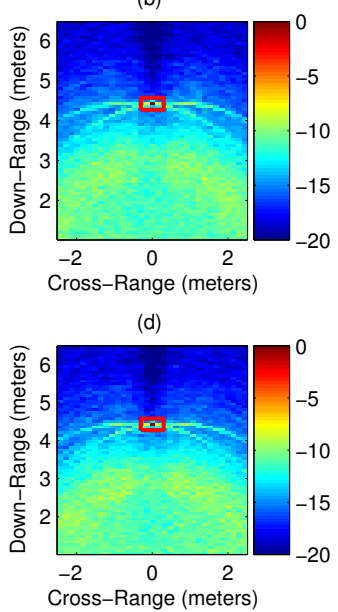

Fig. 3. Backprojection images after spatial filtering is applied to the recovered data from the range profiles: (a) Classic OMP with $20 \%$ random frequencies, (b) Classic OMP with $40 \%$ random frequencies, (c) Modified OMP with $20 \%$ random frequencies, (d) Modified OMP with $40 \%$ random frequencies

the SVD based method (Fig. 2(b)) to the full dataset of 728 frequencies and 67 antenna locations. We observe that both methods suppressed the front wall return and unmasked the target. As discussed in the introduction, the entire frequency band may not always be available. Combined with the desire for fast data acquisition, this would result in a different set of frequencies being used at different antenna positions, leading to different wall reflection phase returns across the antennas and rendering both spatial filtering and SVD methods ineffective.

The proposed method solves this shortcoming by recovering all the frequency measurements at each antenna location through the $l_{1}$ norm range profile reconstruction. Both classic OMP and modified OMP were used to recover range profiles at each antenna location using only $20 \%$ of the frequency measurements. Fig. 3(a) and Fig. 3(c) show the backprojection images obtained after applying spatial filtering to the full data recovered from the reconstructed range profiles using classical OMP and modified OMP, respectively. Each imaged pixel is the result of averaging 100 runs, with different random frequency selections for each run. We observe that the target has not been localized in both images. Next, the number of

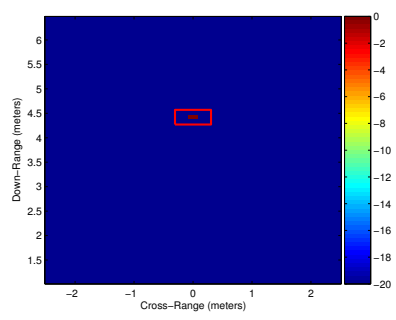

Fig. 4. $l_{1}$ minimization based scene reconstruction using the range profiles obtained with modified OMP with $40 \%$ frequencies.

Table 1. TCR: Simulated Data

\begin{tabular}{|c|c|c|}
\hline$\%$ of Frequencies & Backprojection & $l_{1}$ reconstruction \\
\hline \hline $40 \%$ Different at each antenna & 4.66 & 341.15 \\
\hline $40 \%$ Same at each antenna & 86.36 & 1300.19 \\
\hline $100 \%$ & 151.36 & 1303.53 \\
\hline
\end{tabular}
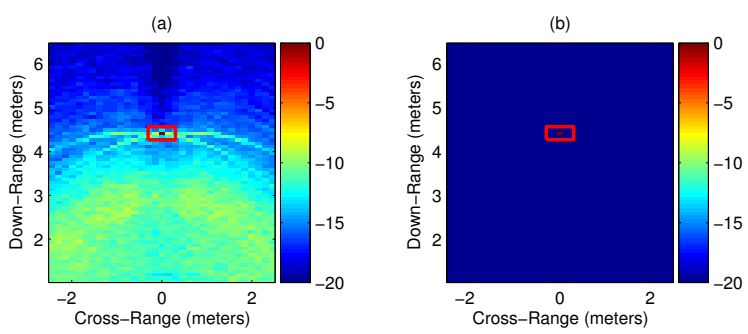

Fig. 5. SVD applied to the recovered data from range profiles obtained using modified OMP with $40 \%$ random frequencies: (a) Backprojection image, (b) $l_{1}$ minimization based scene reconstruction.

frequencies used for range profile reconstruction was increased to $40 \%$ and the corresponding backprojection images for classic OMP and modified OMP are provided in Fig. 3(b) and Fig. 3(d), respectively. The classic OMP required 87 iterations $(87 d \approx 6.4)$ to ensure that all the range bins corresponding to the region of interest were scanned. With the modified OMP, the number of iterations reduced to 59 as a consequence of removing all range bins from 0 to $2 \mathrm{~m}$ in the first iteration. Therefore, exploitation of prior information about the wall location resulted in a $32 \%$ reduction in the number of iterations required by OMP.

Finally, Fig. 4 shows the image obtained using $l_{1}$ minimization with the full frequency data recovered from the reconstructed range profiles using modified OMP with $40 \%$ random frequencies. Comparing Fig. 4 and Fig. $3(\mathrm{~d})$, we observe that the $l_{1}$ reconstruction provides a less cluttered image compared with backprojection.

As a performance measure, we use the Target-to-Clutter Ratio (TCR) [9], which is defined as the ratio between the maximum pixel magnitude value of the target to the average pixel magnitude value in the clutter region. The latter excludes the wall clutter. Table 1 summarizes the TCR results obtained in this section. In general, the TCR is im- 



Fig. 6. TWRI images of the experimental scene: (a) no preprocessing, (b) after background subtraction.


Fig. 7. Wall mitigation techniques with full data: (a) Spatial filtering, (b) SVD approach.

proved when using $l_{1}$ reconstruction over backprojection. This ratio assumes 4.66 in Fig. 3(d), whereas it takes the value of 341.15 in Fig. 4. Further, using the full data set provides superior results to reduced data set, irrespective of whether $l_{1}$ norm reconstruction method or backprojection technique is used.

Similar results were obtained with the SVD approach as shown in Fig. 5.

\section{EXPERIMENTAL RESULTS}

A Through-the-Wall SAR system was set up in the Radar Imaging Lab at Villanova University. The signal and system parameters were chosen to be the same as those used for the simulated data. A vertical dihedral was used as a target and was placed at $(0,4.43) \mathrm{m}$, behind a $0.14 \mathrm{~m}$ thick solid concrete block wall. The size of each face of the dihedral is $0.39 \mathrm{~m}$ by $0.28 \mathrm{~m}$. The empty scene without the dihedral target present was also measured.

Fig. 6(a) shows the image obtained by applying backprojection to the full raw data without any preprocessing, wherein the wall returns masked the target behind the wall. The true position of the dihedral is indicated in the figure with a red rectangle. For comparison, the image obtained after background subtraction is provided in Fig. 6(b), which clearly shows the target.

If all the frequency measurements are available, spatial filtering and SVD approach produce the backprojection images shown in Figs. 7(a) and 7(b), respectively. Although the wall return has not been completely suppressed, its shadowing effect has been reduced thereby allowing the detection of the target.

Fig. 8 shows the backprojection images obtained after applying spatial filtering to the data recovered from the (a)



(c)

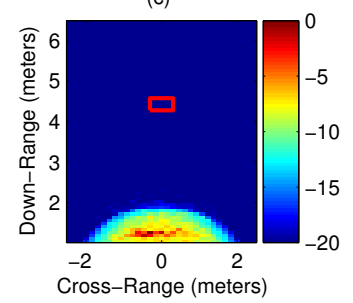

(b)

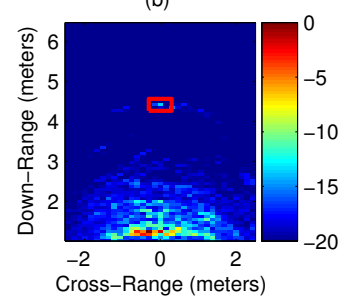

(d)

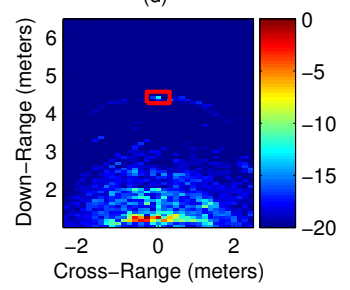

Fig. 8. Backprojection images after spatial filtering is applied to the recovered data from the range profiles: (a) Classic OMP with 20\% random frequencies, (b) Classic OMP with $40 \%$ random frequencies, (c) Modified OMP with $20 \%$ random frequencies, (d) Modified OMP with $40 \%$ random frequencies

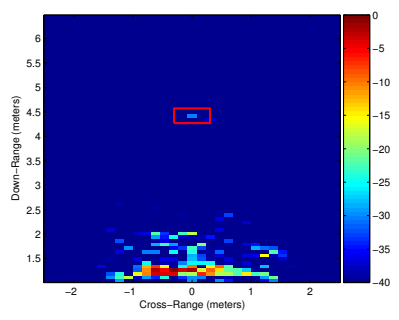

Fig. 9. $l_{1}$ minimization based scene reconstruction using the range profiles obtained with modified OMP with $40 \%$ frequencies.

range profiles using fewer frequencies. Similar to the simulated data case, Fig. 8(a) and Fig. 8(c) are based on random selection of $20 \%$ frequencies for range profile reconstruction using classical OMP and modified OMP, respectively, while Fig. 8(b) and Fig. 8(d) consider $40 \%$ randomly selected frequency samples for range reconstruction using classical OMP and modified OMP. The target is visible in Fig. 8(c) and Fig. 8(d) only.

Finally, Fig. 9 shows $l_{1}$ minimization based scene reconstruction using the full frequency data recovered from the estimated range profiles. In Fig. 9, different sets of $40 \%$ randomly chosen frequencies are used at each antenna position and the modified OMP is used to obtain each range profile. Comparing with the corresponding backprojection image, we observe that the $l_{1}$ reconstruction is less cluttered.

The image quality is compared in Table 2 by means of TCR [9], where the cost for not having all frequency measurements available is clearly shown. While Fig. 7(a) provides a TCR equal to 8.52 , the backprojection image obtained using fewer frequencies, shown in Fig. 8(d), is equal to 2.63. On the other hand, having the same set 
Table 2. TCR: Experimental Data

\begin{tabular}{|c|c|c|}
\hline$\%$ of Frequencies & Backprojection & $l_{1}$ reconstruction \\
\hline \hline $40 \%$ Different at each antenna & 2.63 & 24.98 \\
\hline $40 \%$ Same at each antenna & 6.41 & 697.41 \\
\hline $100 \%$ & 8.52 & 1096.49 \\
\hline
\end{tabular}

of frequencies at each antenna position is shown to improve the performance of the $l_{1}$ reconstructed image quality as compared with using different frequency sets. The $l_{1}$ minimization based scene reconstruction after application of the SVD approach to the recovered range profiles (not shown here) provides almost identical performance to Fig. 9.

\section{CONCLUSIONS}

In this paper, we proposed a technique for mitigating the strong clutter introduced by the front wall, which prevents the direct application of compressive sensing for stationary scene reconstruction in TWRI. A CS approach is used to first reconstruct the sparse range profiles. The Fourier transform applied to the recovered range profiles provides the signal responses of all frequencies, thus allowing signal processing techniques, such as spatial filtering and subspace projections, to capture and remove the wall EM returns. The proposed method is robust in the sense that it permits the use of different sets of frequencies at each antenna location, which becomes a requirement in circumstances where some individual frequencies or frequency subbands may be unavailable due to competing wireless services or intentional interferences.

\section{REFERENCES}

[1] M. G. Amin, "Through-the-Wall Radar Imaging," CRC Press, 2010.

[2] M. G. Amin and F. Ahmad, "Wideband Synthetic Aperture Beamforming for Through-the-Wall Imaging," IEEE Signal Processing Magazine, vol. 25, no. 4, pp. 110-113, Jul, 2008.

[3] F. Ahmad, M. G. Amin, and P. D. Zemany, "DualFrequency Radars for Target Localization in Urban Sensing," IEEE Trans. Aerospace and Electronic Systems, vol. 45, no. 4, pp. 1598-1609, Oct, 2009.

[4] C. Debes, M. G. Amin, and A. M. Zoubir, "Target Detection in Single-and Multiple-View ThroughThe-Wall Radar Imaging," IEEE Trans. Geoscience and Remote Sensing, vol. 47, no. 5, pp. 1349-1361, May, 2009.

[5] Y.S. Yoon and M. G. Amin, "High-Resolution Through-The-Wall Radar Imaging Using Beamspace MUSIC," IEEE Trans. Antennas and Propagation, vol. 56, no. 6, pp. 1763-1774, Jun, 2008.
[6] C. Thajudee, W. Zhang, and A. Hoorfar, "TimeDomain Wall Parameter Estimation and Mitigation for Through-the-Wall Radar Image Enhancement," Progress In Electromagnetics Research Symposium, Cambridge, USA, Jul, 2010.

[7] M. Dehmollaian and K. Sarabandi, "Refocusing Through Building Walls using Synthetic Aperture Radar," IEEE Trans. Geoscience and Remote Sensing, vol. 46, no. 6, pp. 1589-1599, 2008.

[8] F.H.C. Tivive, A. Bouzerdoum, and M.G. Amin, "An SVD-Based Approach for Mitigating Wall Reflections in Through-the-Wall Radar Imaging," IEEE Int. Radar Conference, Kansas City, USA, pp. 519524, May, 2011.

[9] Y.S. Yoon and M. G. Amin, "Spatial Filtering for Wall-Clutter Mitigation in Through-the-Wall Radar Imaging," IEEE Trans. Geoscience and Remote Sensing, vol. 47, no. 9, pp. 3192-3208, 2009.

[10] E. Lagunas, M. Amin, F. Ahmad, and M. Najar, "Wall Mitigation Techniques for Indoor Sensing within the Compressive Sensing Framework," submitted to IEEE Sensor Array and Multichannel Signal Processing Workshop, Hoboken, USA, Dec, 2011.

[11] Y.S. Yoon and M. G. Amin, “Through-the-Wall Radar Imaging Using Compressive Sensing Along Temporal Frequency Domain," IEEE Int. Conf. Acoustics, Speech and Signal Processing (ICASSP), Dallas, USA, Mar, 2010.

[12] F. Ahmad and M. G. Amin, "Noncoherent Approach to Through-The-Wall Radar Localization," IEEE Trans. Aerospace and Electronic Systems, vol. 42, no. 4, pp. 1405-1419, Oct, 2006.

[13] E. J. Candes and M. B. Wakin, "An Introduction to Compressed Sampling," IEEE Signal Processing Magazine, vol. 25, no. 2, pp. 21-30, Mar, 2008.

[14] S. S. Chen, D. L. Donoho, and M. A. Saunders, "Atomic Decomposition by Basis Pursuit," SIAM Journal on Scientific Computing, vol. 20, no. 1, pp. 33-61, 1999.

[15] Y.S. Yoon and M. G. Amin, "Compressed Sensing Technique for High-Resolution Radar Imaging," Proc. SPIE, vol. 6968, pp. 69681A-1-69681A-10, 2008.

[16] J. A. Tropp and A. C. Gilbert, "Signal Recovery From Random Measurements Via Orthogonal Matching Pursuit," IEEE Transactions on Information Theory, vol. 53, no. 12, pp. 4655-4666, Dec, 2007. 